\title{
DESCENDANTS OF REGULAR LANGUAGE IN A CLASS OF REWRITING SYSTEMS : ALGORITHM AND COMPLEXITY OF AN AUTOMATA CONSTRUCTION .
}

\author{
M.Benois \\ L.S.D. , I.M.A.G. , Grenoble 1 University , \\ B.P.68, 38402 St Martin d'Hères Cedex
}

Introduction :

Recent works on public key encryption for secure network communication [7] have brought back the following problem : given a regular set $R$ on $A^{*}$, defined by a non deterministic finite automaton with $n$ states and a rewriting system $T$, how can we construct an automaton that recognizes the set of descendants of $R: \Delta^{*}(R)$ when this language is regular [1]. Some algorithms are found by Book and Otto [6] or Sakarovitch and me [3], in very particular cases of systems and gave complexity in $O\left(n^{4}\right)$ in [6] and $O\left(n^{3}\right)$ in [3]. Here we give a strong extension of these algorithms in a large class of systems however the complexity of our algorithm does not depend on the lenght of the words of $T$ and is at most in $O\left(n^{6}\right)$.

1. Semi Thue systems : definitions and results .

Given a finite subset $T$ of $A^{*} x A^{*}$, we can consider it either as a non symetric system and its elements $(f, g)$ as rewriting rules, or as a symetric system which generates a congruence and which may be Church Rosser. In this work we study systems as rewriting rules and only in the conclusion we transform the results in the Church Rosser view point. Below some definitions and properties of the studied systems are given.

Let $A$ be a finite set, $A^{*}$ is the free monoid generated by $A$ with the empty word 1 as identity. The lenght of a word $f$ of $A^{*}$ is denoted by $|f|$. A Thue system $T$ is a finite subset of $A^{*} X A^{*} ; T$ defines a regular relation denoted by $\rightarrow T,[9]$ and defined by :

$u \rightarrow T \vee$ iff there exist $x$ and $y$ in $A^{*}$ and $(f, g)$ in $T$ such that $u=x f y$

and $v=x g y$.

The transitive ( resp. transitive and reflexive) closure of $\rightarrow T$ is denoted by $\rightarrow^{+} T\left(\right.$ resp. $\left.\rightarrow^{*} T\right)$. A word $v$ is a descendant of a word $u$ in $T$ when 
$u \rightarrow^{+} T v$ and $\Delta^{+}(u)$ is the set of all the descendants of $u$ and $\Delta^{*}(u)=\Delta^{+}(u) \cup\{u\}$. A T-chain $u_{1}, u_{2}, \ldots u_{n}$ is a sequence of words such that $\forall i u_{i} \rightarrow u_{i+1}$.

Let's recall some well known definitions:

-a word is irreducible for $T$ if there exists no T-chain beginning with it .

$-T$ is noetherian if every $T$-chain is finite. This implies that $\forall u$ in $A^{*}, \Delta^{*}(u)$ is finite, [9].

and give some new ones:

-Two systems $T$ and $T$ are equivalent if the their generated relations $\rightarrow^{*} T$ and $\rightarrow^{*} T$ are equal. Then the sets of descendants of a word $u$ are equal.

- Two systems $T$ and $T$ are confluently equivalent if : whenever $u \rightarrow{ }^{*} T^{\prime}\left(\right.$ resp. $\left.u \rightarrow{ }^{*} T^{\prime} u^{\prime}\right)$ then $\exists v$ such that $u \rightarrow{ }^{*} T^{v}$ and $u^{*} \rightarrow{ }^{*} T^{\prime} v$ ( resp. $u \mapsto^{*} T v$ and $u^{\prime} \rightarrow^{*} T v$ ). This relation between systems is an equivalence when the systems are confluent .

It is clear that two equivalent systems are confluently equivalent and that the contrary is false. The decidability of the equivalence problem is implied by the decidability of the following particular word problems : given iwo words $u$ and $v$, is one of them, a descendant of the other, for T and T'.

Proposition 1.1 : If $\mathrm{T}$ and $\mathrm{T}^{\prime}$ are two equivalent systems, they are noetherian at the same time .

Now let us consider the properties of words which describe a system T and denote by :

$$
\begin{aligned}
& -H=\left\{f \in A^{*}, \exists g \in A^{*} g \neq 1 \text { and }(f, g) \in T\right\} \\
& -N=\left\{f \in A^{*}, \exists g \neq 1 \text { and }(f, g) \in T\right\} \\
& -C=\left\{g \in A^{*}, g \neq 1 \text { and }(f, g) \in T\right\}
\end{aligned}
$$

in certain cases may be HUN $\neq \varnothing$.

The properties of our systems will be defined below:

- $T$ is basic if words in $C$ do not overlap properly words in HUN, that is : $\forall \mathfrak{f} \in \mathrm{H} \cup N, \forall \mathrm{g} \in \mathrm{C}$ if $\exists \mathrm{u}, v, w$, such that either $\mathrm{f}=\mathrm{uv}$ and $\mathrm{g}=\mathrm{vw}$ then $\mathrm{w}=\mathrm{f}$ 
or $f=v u$ and $g=w v$ then $w=1 ;[12]$ and $[13]$.

- $T$ is semi-reduced if a factor of a word in $\mathrm{C}$ is never in HUN .Or every word in $C$ is irreducible; [11].

- $\mathrm{T}$ is reducible if $\mathrm{T}$ is equivalent to a semi-reduced system.

- $T$ is confluently reducible if $T$ is confluently equivalent to a semi-reduced system.

The following propositions explain the aim of these definitions:

Proposition 1.2 : A basic and semi-reduced system is noetherian .

The proof goes by induction on the difference between the lenght of a word and the lenght of its factors in HUN. A useful consequence of proposition 1.2 is that in semi-reduced basic system $\forall \mathrm{u} \in A^{*}, \Delta^{*}(u)$ is finite

Proposition 1.2 : One can decide if a given system $T$ is confluently reducible.

This implies that if $\mathrm{T}$ is noetherian then $\mathrm{T}$ is confluently reducible .

2. Properties of sets related to a system $T$ on $A$.

We omit the $T$ in this part since $T$ is always the same .Notations:

$-\Delta^{1}(u)=\left\{v \in A^{*}\right.$ such that $\left.u \rightarrow v\right\}$.

$-\Delta^{i}(u)=\left\{v \in A^{*}\right.$ such that $\exists w \in \Delta^{i-1}(u)$ and $\left.w \rightarrow v\right\}$.

$-\Delta_{\#}(u)=\left\{v \in A^{*}\right.$ such that $\exists n$ and $\left(f_{1}, g_{1}\right),\left(f_{2}, g_{2}\right), \ldots\left(f_{n}, g_{n}\right) \in T$ and

$x_{1}, x_{2}, \ldots x_{n+1} \in A^{*}$ and $\left.u=x_{1} f_{1} x_{2} f_{2} \ldots f_{n} x_{n+1}, v=x_{1} g_{1} x_{2} g_{2} \ldots g_{n} x_{n+1}\right\}$.

These definitions are extented to a set of words instead of a word and:

$$
\begin{aligned}
& -\Delta^{i}(P)=U_{U \in P} \Delta^{i}(u) \\
& -\Delta^{+}(P)=U_{i \geq 1} \Delta^{i}(P), \Delta^{*}(P)=U_{i \geq 0^{\prime}} \Delta^{i}(P) .
\end{aligned}
$$

We have the following properties: 
If $P \subset P^{\prime}$ then $\triangle(P) \subset \Delta\left(P^{\prime}\right), \Delta_{\#}(P) \subset \Delta_{\#}\left(P^{\prime}\right), \Delta^{*}(P) \subset \Delta^{*}\left(P^{\prime}\right)$;

- $P \subset \Delta^{*}(P), \Delta^{+}\left(\Delta^{+}(P)\right)=\Delta^{+}(P), \Delta^{*}\left(\Delta^{+}(P)\right)=\Delta^{+}(P)$;

$-\Delta(P) \subset \Delta_{f \neq}(P) \subset U_{i \leq \max |u|, u \in P} \Delta^{i}(P)$;

$-\Delta_{\#}(P)=\Delta^{+}(P), \Delta_{\#}^{*}(P)=\Delta^{\star}(P)$;

-These operators are stable for union and intersection of subsets.

3. Descendants of regular languages in a semi-reduced basic system $T$.

The set of semi-reduced basic systems is a large extension of the well known and studied following systems:

-special systems where $H=\varnothing$

-monadic systems where $\mathrm{C} \subset \mathrm{A}$.

They are not all lenght-reducing, however when they are not, $\Delta^{*}(U)$ remains finite ,by proposition 1.2 .

We refer to Hopcropft and Ullman [10] for the definitions of finite automata and of regular languages on $A$ and we follow their notations. Given a regular language $R$ on $A$ accepted by a non deterministic finite state automaton $A=\left(Q, A, q_{0}, \partial, F\right)$, we want to transform this automaton in another that recognizes all the descendants of $R: \Delta^{*}(R)$ with respect to a semi-reduced, basic system $T$. The algorithm we describe and prove below is also proof of:

Theorem 1 : The set $\Delta^{*}(R)$ of the descendants in a semi-reduced basic system of a regular language $\mathrm{A}$ is a regular language on $\mathrm{A}$.

The study of the complexity of the algorithm gives the next theorem:

Theorem 2: Let $T$ be a finite semi-reduced basic system on A and $R$ be a regular language on A specified by a non deterministic finite automaton with $n$ states, one can effectively construct in $O\left(n^{6}\right)$ steps a non deterministic finite automaton that recognizes $\Delta^{*} T(R)$.

This theorem generalizes the algorithm described in [3] and its complexity in some particular cases is:

$-O\left(n^{4}\right)$ if $H=O$.

$-O\left(n^{4}\right)$ if $T$ is monadic (i.e. for every $\left.g \in C|g| \leq 1\right)$.

$-O\left(n^{4}\right)$ if for every $f \in H U N \quad|f| \leq 2$. 
$-O\left(n^{3}\right)$ if for every feHUN $|f| \leq 2$ and for every $g \in C|g| \leq 1$.

4. The basic principles of computing an automaton that recognizes $\Delta^{*}(R)$.

First we will consider the given automaton of $=\left(Q, A, q_{0}, \partial, F\right)$ as a directed labelled graph $G(J t)$ the vertices of which are elements of $Q$. Its edges represent the transition function $\partial:\left(q, x, q^{\prime}\right)$ is an edge from $q$ to $q^{\prime}$ with label $x \in A$ iff $q^{\prime} \in \partial(q, x)$; a vertex $q_{0}$ is the initial state and $F$ is a subset of vertices. A path of $G(0 t)$ is a sequence of vertices and edges denoted by $\left(q, u, q^{\prime}\right)$ where $q$ is the beginning, $q^{\prime}$ the end and $u$ is the concatenation of the labels of the edges. A word $u$ is recognized by of iff a path $\left(q_{0}, u, q^{\prime}\right)$ exists in $G(\mathfrak{f})$ with $q^{\prime} \in F$.

The main idea is : for every feHUN and every path $\left(q, f, q^{\prime}\right)$ in $G(f t)$ we have to add a new path $\left(q, g, q^{\prime}\right)$ for every $g$ such that $(f, g) \in T$. This implies that we add new vertices and new edges and so create new paths $\left(q, f, q^{\prime}\right)$ and the process may be infinite. We choose a particular way to add these new vertices and edges; it is easy to prove that in this way the process is finite but we have then to prove that the graph we construct recognizes $\Delta^{*}(R)$.

Initially $G_{0}$ is the graph of of that recognizes $R$, its vertices will be called initial vertices. The finiteness of the algorithm comes from the consideration of a queue ARC which, at the beginning, countains all the edges of $G_{0}$ labelled by a letter of an $f \in F U N$; each step of the algorithm can put new elements is this queue.

For every edge $(i, x, j)$ at the front of the queue and every $f \in H \cup N$ we look, in the graph constructed at this step, for the paths $\left(q, f, q^{\prime}\right)$ which contain the edge $(i, x, j)$, this is part one in the algorithm. Whenever we found such a path we have two ways in adding new paths :

-if $f \in \mathrm{H}$ we add, first new vertices and new edges related to these vertices to realise a path $\left(q, g, q^{\prime}\right)$ for every $g$ such that $(f, g) \in T$; then the first new edge created in the queue ARC. This is part two of the algorithm.

-if $f \in N$ we add, first a new edge $\left(i, y, q^{\prime \prime}\right)$ every time $\left(j, y, q^{\prime \prime}\right)$ is already an edge in the graph, then this new edge in the queue ARC.

This is part three of the algorithm .

To remember which paths we have already added, we consider $|\mathrm{C}|+1$ Boolean matrices,one for $f \in N$ denoted $U N I$ and the others for $g \in C$ denoted $\operatorname{MEM}(g)$ the sizes of which will be defined later.

For every new vertex $s$ constructed in part two it is convenient to 
remember its generating vertices $O R(s)$ and $\operatorname{EXT}(s)$, the suffix $R D(s)$ which labels the path (s,EXT(s)) and the prefix $R G(s)$ that labels the path (OR(s), s).

In order to prove, that we have to consider a finite number of paths $\left(q, f, q^{\prime}\right)$, we use the chosen properties of the system $T$ and establish the following:

Lemma 4.1: in every state of the graph constructed by the algorithm, the outdegree of a new vertex is exactly 1 , the indegree of a new vertex is 1 except for the successor of an initial vertex but then, every inedge has the same label.

The proof goes by induction on the creation time of the vertices and uses the property: $T$ is basic.

Corollary 4.1 : When a path contains a non initial vertex s created by a path $(q, f, q)$ the vertices of which are, $s_{1}, s_{2}, \ldots s_{|g|-1}$, it contains the subpath of $\left(q, f_{,} q^{\prime}\right):\left(s_{1}, s_{i}\right)$ if $s_{j}$ is its end, otherwise $\left(s_{1}, q^{\prime}\right)$.

This corollary and the property: $T$ is semi-reduced, prove:

Proposition 4.1 : Whenever $\left(q, q^{\prime}, q^{\prime}\right)$ is a path of a graph in the algorithm and $f \in F U N, q$ and $q^{\prime}$ are initial vertices.

Corollary 4.2 : The algorithm adds at most $|\mathrm{C}| \cdot \mathrm{n}^{2} \cdot \max _{\mathrm{g} \in \mathrm{C}}(|\mathrm{g}|-1)$ vertices to the initial graph.

Therefore we can consider one set $W$ of $m=|C| \cdot n^{2} \cdot \max _{\mathrm{g} \in \mathrm{C}}(|\mathrm{g}|-1)$ vertices, the initial vertices are a subset $S,|S|=n$ and $m$ is in $O\left(n^{2}\right)$. The algorithm only adds edges. The queve ARC will contain at most $|A| \cdot \mathrm{m}^{2}$ edges. New terms are put in ARC when new paths $\left(q, f, q^{\prime}\right)$ are found and new coefficients 1 in matrices UNI and MEM(g) the size of which are $n$, hence the algorithm has a finite number of steps.

5. Description of the algorithm.

Data : 
The system $\mathrm{T}$ is defined by the sets $\mathrm{H}, \mathrm{N}, \mathrm{C}$ and -for every $f \in H$, a set PROJ $(f)=\{g$ such that $(f, g) \in T\}$ -for every $x \in A$, a set $F A C T(x)=\{f \in H U N$ such that $x$ is a letter of $f\}$ -for every $f \in F A C T(x)$, a set of couples of words

$D E C(f, x)=\{(F G, F D)$ such that $F G \times F D=f\}$ -for every $g \in C$ an integer $\mathrm{l}(\mathrm{g})=|\mathrm{g}|-1$.

The graphs are defined by a set of vertices $\{1,2, \ldots \mathrm{m}\}$ where $S=\{1,2 . n\}$ and $|\mathrm{A}| \mathrm{m} . \mathrm{m}$ Boolean matrices:

$$
\operatorname{MAT}(x)(i, j)=1 \text { iff }(i, x, j) \text { is an edge. }
$$

Initially we consider all the edges of $G_{0}$. The set $F$ of final vertices is initially the set of final states of ot .

We have also the auxillary data :

-a $n . n$ Boolean matrix UNI, initially $U N I(i, j)=1$ iff $i=j$. -for every $\mathrm{g} \in \mathrm{C}$ a $n . n$ Boolean matrix $\mathrm{MEM}(\mathrm{g})$ initially $O$. -for every $s>n$ two elements of $S: O R(s)$ and EXT(s), and two words $R G(s)$ and $R D(s)$, initially they are $\varnothing$ or 1 . -a counter COMPT : an integer $\geq n$ used for the allocation of the new edges on "new" vertices. Initially COMPT $=n$.

-a counter ETAP of stages of the algorithm related to a state of the graph and the order of the appearence of 1 in the matrices UNI and $\operatorname{MEM}(\mathrm{g})$.

-a queue ARC initially contains all the edges of $G_{0}$.

Part one of the algorithm :The search of paths $\left(q, f^{\prime}, q^{\prime}\right)$ that contain a given edge $(i, a, j)$.

We choose, for this graph algorithm ,to use a m.k matrix, the lines of which construct the sucessor of the last line, and we short our search whenever a vertices $s>n$ by putting directly its path-successor by corrollary 4.1.The result is a set $Q x Q^{\prime}$ of all the couples of extremities of such a path . We search $Q$ then $Q^{\prime}$ and have to compute their cartesian product. Roughly the search of $Q^{\prime}$ can be describe by :

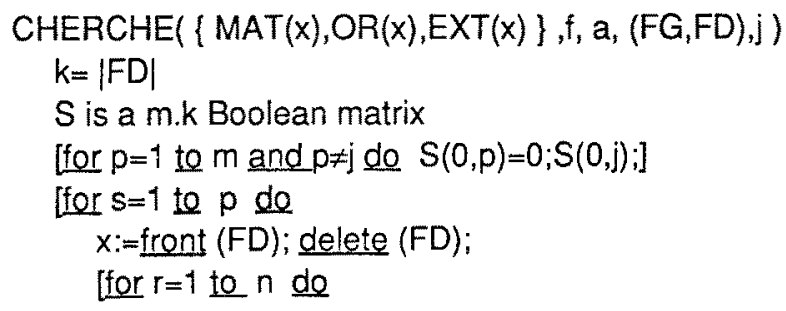


if $S(s-1, t)=1$ then [for $t=1$ to $m$ do if $\operatorname{MAT}(x)(r, t)=1 ; S(s, t)=1$;] [for $r=n+1$ te $m$ de

if $S(s-1, r)=1$ then compare (FD and $\mathrm{RD}(r)$ ); if $R D(r)$ prefix of $F D$ then $S(S+|R D(r)|-1, E X T(r))=1 ;]]$

We denote part one by: $\operatorname{CHERCHE}\left((i, a, j),\left\{,(F G, F D), Q \times Q^{\prime}\right)\right.$.

Part two of the aigorithm: The adjunction of a path $\left(q, g, q^{\prime}\right)$ whenever a path $\left(q, f, q^{\prime}\right)$ is found by part one with $f \in H$; and the adjunction of one edge in ARC .

We choose to put the extremities of new edges with respect to the order of the numbers that represent the vertices $n+1, n+2, \ldots, m$ by the mean of the counter COMPT. We denote this part by:

$\operatorname{ADJ}\left(\left(q, q^{\prime}\right), g,\{\operatorname{MAT}(x), O R(x), \operatorname{EXT}(x), R G(x), R D(x), A R C)\right.$

and describe it roughly:

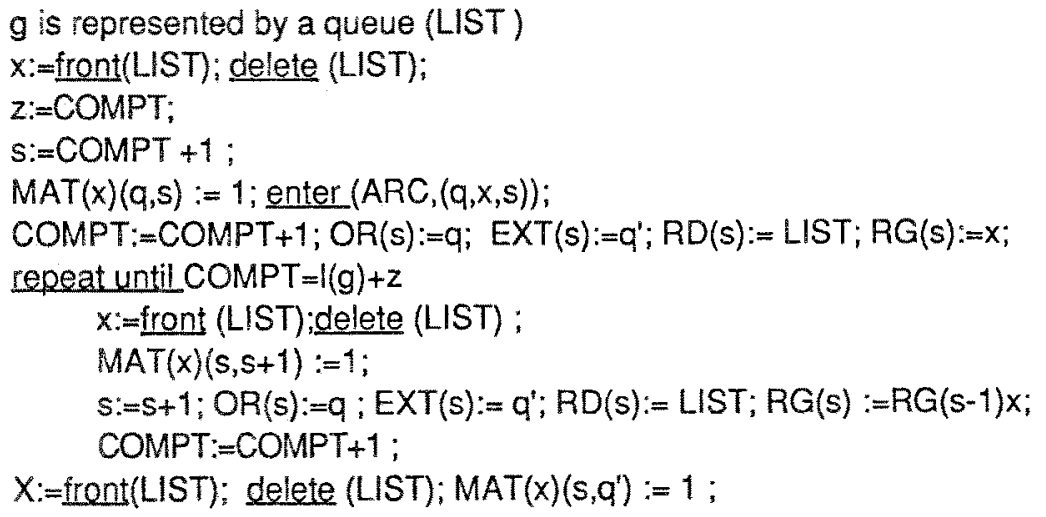

Part three of the algorithm :The adjunction of edges $(q, x, r)$, whenever a path $\left(q, f, q^{\prime}\right)$ is found in part one with $f \in N$ and $\left(q^{\prime}, x, r\right)$ is already an edge of the graph; these edges are added both in the grah and in ARC .

We denote this part by $\operatorname{CLOT}\left(\left(q, q^{\prime}\right), x, \operatorname{MAT}(x), A R C\right)$ :

[for $s=1$ 1 $10 \mathrm{~m}$ do

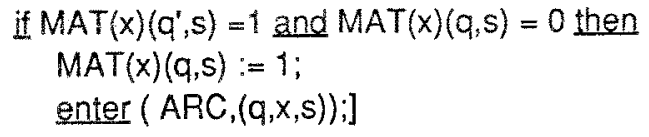


The whole algorithm can be now simply describe :

$$
\text { ETAP: }=1
$$

$B C:$ if empty (ARC) then halt :

$(i, a, j):=$ front $A R C$; delete $(A R C)$;

[for all $f \in F A C T$ (a) do

[for all $(F G, F D) \in D E C(f, a)$ do

CHERCHE((i,a, $\left.), f,(F G, F D), Q \times Q^{\prime}\right)$;

ffor all $\left(q, q^{\prime}\right) \in Q \times Q^{\prime}$ do

if $f \in N$ and $U N I\left(q, q^{\prime}\right)=0$ then

ETAP:=ETAP+1;

UNI $\left(q, q^{\prime}\right):=1$;

if $q^{\prime} \in F$ then $F:=F \cup\left\{q^{\prime}\right\}$;

[for all $x \in A$ do CLOT $\left.\left(\left(q, q^{\prime}\right), x, M A T(x), A R C\right) ;\right]$

else if $f \in H$ then

[for all $g \in P R O J(f)$ do

if $M E M(g)=0$ then

ETAP:=ETAP+1 ;

$\operatorname{MEM}(g)\left(q, q^{\prime}\right):=1$

go to $B C$;

$\left.\left.\left.\left.\operatorname{ADJ}\left(\left(q, q^{\prime}\right), g,\{\operatorname{MAT}(x), O R(x), \operatorname{EXT}(x), R G(x), \operatorname{RD}(x)\}, A R C\right) ;\right]\right]\right]\right]$

6. The running time of this algorithm .

We evaluate each part of the algorithm:

-the lenght of the queue ARC is already in $O\left(n^{4}\right)$ but we can give a smaller bound : initially there are $n^{2}$ edges in ARC the new ones have their invertices in $S$ so the size of ARC is in

$$
O\left(n^{2}\right)+O(n \cdot m)=O\left(n^{3}\right) \text {. }
$$

-generally, part one countains $n . m$ executions of instruction in $O(1)$ and $m-n$ also in $O(1)$; its running time is $O(n \cdot m)+O(m-n)=O\left(n^{3}\right)$. If $H=\varnothing$ then $n=m$ and it becomes $O\left(n^{2}\right)$; if the lenght of words in FUN is smaller than $2, O(n)$. This part is called every time a new edge is put in front of $A R C$, at most $O\left(n^{3}\right)$ times.

-part two $A D J$ has a running time $O(1)$ and is called every time a new 1 appears in $M E M(g)$, at most $|C| \cdot n^{2}=O\left(n^{2}\right)$ times.

-in part three each instruction costs $O(1)$ and is called $m$ times, the running time is $\mathrm{O}\left(n^{2}\right)$. This part is called when a new 1 appears in 
UNI, at most $n^{2}$ times.

Then the running time of the whole algorithm is:
-in general
-when $\mathrm{H}=\varnothing$
$O\left(n^{3}\right) O\left(n^{3}\right)+O(1) O\left(n^{2}\right)+O\left(n^{2}\right) O\left(n^{2}\right)=O\left(n^{6}\right)$
$\mathrm{O}\left(\mathrm{n}^{2}\right) \mathrm{O}\left(\mathrm{n}^{2}\right)+$
$O(n) O\left(n^{2}\right)=O\left(n^{4}\right)$
-if max $|g| \leq 1$ (monadic) $O\left(n^{2}\right) O\left(n^{2}\right)+O(1) O\left(n^{2}\right)+O(n) O\left(n^{2}\right)=O\left(n^{4}\right)$
-if max $|f| \leq 2$, fEFUN $\left.O(n) O\left(n^{3}\right)+O(1) O\left(n^{2}\right)+O n^{2}\right) O\left(n^{2}\right)=O\left(n^{4}\right)$
if $\max |g| \leq 1$ and $\max |f| \leq 2, f \in H \cup N \quad O(n) O\left(n^{2}\right)+O(1) O\left(n^{2}\right)+O(n) O\left(n^{2}\right)=O\left(n^{3}\right)$

7.The automaton represented by the last state $G_{t}$ of the algorithm graph recognizes $\Delta^{*}(R)$.

Let $G_{i}$ denotes the state of the graph just before the value of ETAP is $i+1, R_{i}$ is the language recognized by the automaton $t_{j}$ the graph of which is $G_{i}$. Eventually $G_{t}=G_{t+1}=G_{t+k}$ when the consideration of the last element of the queue does not create either new value 1 in the matrices UNI and MEM(g) or new edges by ADJ or CLOT. It is easy to prove

Proposition 7.1: $R_{\mathrm{t}} \subset \Delta^{*}(R)$.

The proof uses, properties of $\Delta_{\#,}, \Delta_{\#}^{*}$ and $\Delta^{*}$ seen in 1 and the following lemma:

Lemma 7.1: $R_{i} \subset R_{i-1} \cup \Delta_{\#}\left(R_{i-1}\right)$.

The proof goes by induction on $k$ defined by: given a path $\left(q_{0}, u, q\right)$ in $G_{i}, k$ is the the number of its edges which are not in $G_{i-1}$ and the proof uses Corollary 4.1 .

To prove that $\Delta^{*}(R) \subset R_{t}$ we have to establish further results on the paths of $G_{t}$.

Lemma 7.2 : Every path in $G_{t}$ ending in $S$ is factorized in subpaths ending in $S$, the first edges of which (its caracteristic edge) are in ARC. 
Lemma $7.2:$ If $(i, \mathrm{f}, \mathrm{j})$ is a path in $\mathrm{G}_{\mathrm{t}}$ with $\mathrm{i}$ and $\mathrm{j}$ in $\mathrm{S}$ then :

-if $\mathrm{f} \in \mathrm{N}$ then $\mathrm{UNI}(\mathrm{i}, \mathrm{j})=1$.

-if $\mathrm{f} \in \mathrm{H}, \forall \mathrm{g} \in \mathrm{PROJ}(\mathrm{f}) \quad \mathrm{MEM}(\mathrm{g})(\mathrm{i}, \mathrm{j})=1$ and $a$ path $(\mathrm{i}, \mathrm{g}, \mathrm{j})$ exists

in $\mathrm{G}_{\mathrm{t}}$.

The proof uses Lemma7.2 and the last caracteristic edge to be put in front of ARC.

Lemma7.3 : If $\mathrm{i}, \mathrm{j}, \mathrm{k} \in \mathrm{S}, \mathrm{f} \in \mathrm{N}, \mathrm{h} \in \mathrm{A}^{+}$and $(\mathrm{i}, \mathrm{f}, \mathrm{j})$ and $(\mathrm{j}, \mathrm{h}, \mathrm{k})$ are paths in $\mathrm{G}_{\mathrm{t}}$ where all the vertices of $(\mathrm{j}, \mathrm{h}, \mathrm{k})$,except its ends, are not in $\mathrm{S}$, then a path $(\mathrm{i}, \mathrm{h}, \mathrm{k})$ exsists in $\mathrm{G}_{\mathrm{t}}$.

The proof goes by induction on the value of ETAP when the caracteristic edge of $(\mathrm{j}, \mathrm{h}, \mathrm{k})$ is created.

Then it is easy to prove that $\Delta^{1}\left(R_{t}\right) \subset R_{t}$ then $\Delta^{*}\left(R_{t}\right) \subset R_{t}$ and $\Delta^{*}(R) \subset \Delta^{*}\left(R_{t}\right) \subset R_{t}$.

8.Applications

If we consider the congruence generated by a basic, semi-reduced, Church-Rosser system, each congruence class is defined by its minimal element and our results imply :

-Monoids finitly presented by such systems have the cross-section property [14] and [8].

-The two problems :

-Do two regular languages have a non zero intersection with a congruence class?

-Does a regular language have a non zero intersection with the set of descendants of another regular language?

are decidable for a basic, semi-reduced, Church-Rosser system .

In conclusion ,we think that, this class of system is the largest one in which Theorems 1 and 2 are true, since Church-Rosser, semi-reduced systems exist which are not basic and in which ,sets of descendants of regular sets are not regular [2] . 


\section{References:}

[1] M.Benois: Parties rationnelles du groupe libre, C.R.Acad.Sci.Paris ser. A 269,1969,1188-1190.

[2] M.Benois et P.Butzbach :Langages algébriques déterministes et transversale rationnelle, Bapport de recherche $n^{\circ} 446$, L.S.D. IMAG 1984.

[3] M.Benois et J.Sakarovitch :On the complexity of some extended word problems defined by cancellation rules, Information Processing Letters à paraître.

[4] J.Berstel :Transductions and context free langages.Teubner 1979 .

[5] R.Book, M.Jantzen and C.Wrathall , Monadic Thue Systems, Theoret. Comput. Sci, 19 (1982) 231-251.

[6] R.V.Book and F.Otto : Cancellatio rules and extended word problems, Information Processing Letters 20,1985,5-11.

[7] D.Dolev and A.Yao: On the security of public key protocols, I.E.E.E. Irans.Information Theory IT $29,1983,198-208$.

[8] S.Ellenberg: Automata, Languages, and Machines Vol A,Academic Press, 1974.

[9] G. Huet : Confluent reductions: Abstract properties and applications to term rewriting systems, J. Assoc. Comp. Mach. 27, 1980 797-821.

[10] J.Hopcroft and J.Ullman : Introduction to Automata Theory, Langages and Computation Addison-Wesley, 1979.

[11] P.Narendran: Church -Rosser and related Thue systems, Report $n^{\circ}$ 84CRD176. General Electric Corporate Research and Development. Schenectady, N.Y. , 1984.

[12] M.Nivat (et M.Benois) : Congruences parfaites et quasi parfaites, Séminaire Dubreil, $25^{\circ}$ année, 1971-72, 7-01-09.

[13] J.Sakarovitch :Description des monoïdes de type fini , Rapport $n^{\circ}$ 80-36 LITP, 1980 .

[14] J.Sakarovitch : Syntaxe des langages de Chomsky Thèse Paris7 1979. 